$(\mathrm{p}=0.03)$ and particularly with a more important radiological progression $(\mathrm{p}=0.03)$ was the LFT.

Conclusion Whatever the method used, indirect immunofluorescence or ELISA, anti-filaggrin do not allow to predict radiological damage in community cases of RA of limited duration.

\section{AB0044 AN OUTSTANDING LATE RHEUMATOID ARTHRITIS IN A PATIENT WITH ACROMEGALIC ARTHROPATHY: A CASE REPORT}

L Özçakar, A Sivri, S Bal. Physical Medicine and Rehabilitation, Hacettepe University Medical School, Ankara, Turkey

10.1136/annrheumdis-2001.1009

\section{Background}

Objectives Arthropathy is a consequence of growth hormone excess in acromegalic patients due to articular cartilage and nearby soft tissue involvement. Rheumatoid arthritis (RA) is, on the other hand, another entity, which may sometimes look alike due to the similar clinical findings. We present this case where these both diseases exist at the same time.

Methods A 63-year old male complaining of joint pain and ankle swelling was evaluated. The arthralgia he described, was mainly in his knees, elbows and shoulders, and exaggerated especially at night. Recently accompanying swelling and erythema in his left ankle and in the left second metacarpophalangeal (MCP) joint did exist. He has been diagnosed as acromegalia for 15 years whereas his symptoms related with the joints came out within the last 6 years. He is somatotropinectomized and has also received radiotherapy for his primary disease. His neck, bilateral wrist, elbow and shoulder joints were involved, thus were painful and limited during normal range of motions. MCP joints being worse than the interphalangeal joints were likewise involved. His left ankle and MCP joints were swollen and red additionally. Laboratory and radiological evaluations comprising complete blood count, liver and kidney function tests, serological tests, direct $\mathrm{x}$-rays of the joints were carried out. His erythrocyte sedimentation rate (ESR) was $93 \mathrm{~mm} / \mathrm{h}, \mathrm{RF}$ : $685 \mathrm{IU} / \mathrm{ml}$. The diagnosis of rheumatoid arthritis other than the concurrent acromegalic arthropathy was confirmed with the radiological and clinical findings according to the ARA criteria. He was then treated with steroids.

Results We considered this case challenging and notifying since an acromegalic patient can easily be misdiagnosed as having RA especially when high ESR and morning stiffness accompanies the case. We think the uncommon coexistence i.e. not mentioned up to date in the literature, may enlighten the still unknown pathogenesis of RA with the probability of growth factors mastering the whole event.

Conclusion

\section{AB0045 PERIPROSTHETIC BONE REMODELLING AND RADIOGRAPHIC FIXATION OF A CEMENTLESS TOTAL HIP ARTHROPLASTY WITH TITANIUM STEM IN RHEUMATOID ARTHRITIS AND OSTEOARTHRITIS PATIENTS}

T Taketa, H Ohnishi, H Turukami, N Okimoto, M Takeda, S Okabe, T Nakamura.

Orthopedics Surgery, University of Occup. and Environ. Health, Kitakyushu, Japan
Background New bone formation around cementless stem after total hip arthroplasty is dependent on implant design, material, and mode of porous coating. These osteogenesis patterns are classified as follows, proximal endosteal bone bridging spot welds, increased cortical thickness (cortical hypertrophy) and thin radiodense lines surrounding the tip of the implant (halo pedestal). However, no reports showed the comparison of bone remodelling patterns surrounding stem among diseases: such as osteoarthritis (OA), rheumatoid arthritis (RA), avascular necrosis and neck fracture.

Objectives The purpose of this study is to clarify whether there are differences of the postoperative bone remodelling pattern around the femoral stem between RA and OA patients.

Methods Total hip arthroplasty with the Mallory-Head cement less titanium prosthesis has been performed since 1992 in our institute. This tapered stem has plasma spray coating in proximal one third of it. RA group included 18 hips (mean age at operation was fifty-five years old and mean duration of follow-up was 4.6 years). OA group included 70 hips (mean age at operation was fifty-eight years old and mean duration of follow-up was 5.2 years). Anteroposterior radiographs of the hip were made at 6 weeks, 3 months, 6 months, one year and every year after the operation. We evaluated the frequency and time of new bone remodelling appearance around the stem such as spot welds, cortical hypertrophy, and pedestal. We defined that the radiographic signs of instability were progressive subsidence or the presence of radiolucent lines around the porous coating (1). To evaluate the differences in both groups, the Mann-Whitney's U test and chi-square test were used. $\mathrm{P}$ values of less than 0.01 were considered significant.

Results In both groups, there was no case, which has radiographic sign of instability. Age and intramedurally canal fill of the stem at the operation and follow-up period were not significantly different between RA and OA groups. In endosteal spot welds, the time of its appearance in RA group (2.7 months after operation) was significantly shorter than that in OA group $(5.2$ months) and the frequency of it did not differ between both groups. However, in halo pedestal, the frequency in RA group $(50 \%)$ was significantly lower than that in OA group (87\%) and the time of its appearance did not differ between both groups. On the other hand, concerning about cortical hypertrophy, time and frequency showed no significant differences between two groups.

Conclusion We revealed that in RA patients, endosteal spot welds appeared significantly earlier than that in OA patients, and the frequency of halo pedestal was significantly lower than that in OA patients.

\section{AB0046 PREVALENCE OF TUBERCULOSIS INFECTION IN RHEUMATOID ARTHRITIS PATIENTS TREATED BY CORTICOSTEROID PULSE THERAPY}

C Di Girolamo, MG Ferrucci, N Pappone, C Crisci. Rehabilitation, "Salvatore Maugeri" Foundation, Telese Terme, Italy

10.1136/annrheumdis-2001.1011

Background Several pathogens have been previously reported in rheumatoid arthritis (RA) patients treated by corticosteroid pulse therapy $(\mathrm{CPT})^{1-3}$ and we recently described a case of upper lobe cavitary tuberculosis in a patient with RA under CPT associated to methotrexate. ${ }^{4}$ 
Objectives In order to establish the prevalence of tuberculosis infection in RA patients undergoing CPT, we analysed medical charts of consecutive patients affected by these disease who underwent this regimen.

Methods All the records were from patients attending the clinic for at least 2 years since the CPT administration and were analysed in order to detect a clinical diagnosis of tuberculosis. Routine blood analysis and a Chest x-ray film was available for all the patients in the period established for the follow-up, even if asymptomatic. At present, 11 females (mean age 57.54 years, range 40-74) have been identified, who took 1 gr of Methyilprednisolone i.v. daily in a 3-day course of CPT. One of them was taking methotrexate, 3 cyclosporin and 4 the combination of cyclosporin and methotrexate; 3 patients was under no DMARD. CPT was already administered in 3 patients no more than once in the previous year.

Results Two patients revealed a tuberculosis infection, characterised by cavitary lung involvement and meningitis respectively; diagnosis was confirmed by standard methods for tuberculosis in both the patients. One patient affected by tuberculosis belonged to the treatment group CPT + methotrexate, while the other suspended methotrexate less than one year before the administration of CPT.

Conclusion This finding underlines the necessity of close monitoring for severe opportunistic infections in rheumatoid patients during treatment with CPT and MTX, but seem to suggest a more prominent role played by CPT with respect to other DMARDs.

\section{REFERENCES}

1 Weusten BLAM, Jacobs JWG, Bijlsma JWJ. Corticosteroid pulse therapy in active rheumatoid arthritis. Semin Arthritis 1993;23:183-92

2 Van Der Veen MJ, Bijlsma JWJ. The effect of methylprednisolone pulse therapy on methotrexate treatment of rheumatoid arthritis. Clin Rheumatol. 1993;12:500-5

3 Boerbooms AMT, Kerstens PJSM, van Loenhout JWA, Mulder J, van de Putte LBA. Infections during low-dose methotrexate treatment in Rheumatoid arthritis. Semin Arthritis Rheum. 1995;24:411-21

4 di Girolamo C, Pappone N, Melillo E, Rengo C, Giuliano F, Melillo G. Cavitary lung tuberculosis in a rheumatoid arthritis patient treated with low-dose methotrexate and steroid pulse therapy. Br I Rheumatol. 1998;37:1136-7

\section{AB0047 PROGRESSION OF RADIOLOGICAL DAMAGE IN PATIENTS WITH RHEUMATOID ARTHRITIS IN CLINICAL IN REMISSION; A PROSPECTIVE TWO YEAR FOLLOW-UP STUDY}

ET Molenaar, AE Voskuyl, HJ Dinant, DP Bezemer, BA Dijkmans. Rheumatology, Vrije Universiteit Medical Centre, Amsterdam, The Netherlands

10.1136/annrheumdis-2001.1012

\section{Background}

Objectives The aim of the present study was to investigate the progression of radiological damage in patients with RA in clinical remission during a follow-up of two years.

Methods RA patients $(\mathrm{n}=187)$, in remission as defined by the ACR/Pinals Criteria were included. Radiographs of hands and feet were made at baseline, and 2 years. Radiological damage was assessed according to the Sharp-van der Heijde method (score ranging from 0-448; (SHS)). SHS at baseline and after two years were compared in the complete cohort as well as in subgroups of patients with persistent remission and those suffering from excacerbations (Wilcoxon test). The following variables, measured at baseline, were assessed as possible riskfactors for radiological progression: number of tender and swollen joints, Ritchie score, Disease Activity Score (DAS), serum titers of IgM and IgA rheumatoid factors, the visual analogue scale (VAS) for the physicians impression of disease activity and the Health Assessment Questionnaire (HAQ) score.

Results At baseline and after two years, the mean SHS of the complete group were 49 and $51(p<0.001)$. In the subgroup of persistant remission, the mean SHS were 52 and 53 (p = 0.004). No significant correlations were found between progression of radiographic damage and baseline variables (rho $<0.1, p$ $>0.1)$. In the subgroup of exacerbation, the mean SHS were 46 and $51(\mathrm{p}<0.001)$. Progression of radiographic damage correlated significantly with the following variables at baseline: SHS (rho 0,40), HAQ-score (rho 0.28), serum IgM-RF (rho 0.29), serum IgA-RF (rho 0.25), but not with the VAS and the DAS.

Conclusion Radiological damage proceeds in RA patients in remission and is not related to disease activity.

\section{AB0048 RHEUMATOID ARTHRITIS IN ACROMEGALIC PATIENT: A CASE REPORT}

V Çobankara, P Aprab, MA Öztürk, Ý Ertenli, S Kiraz, M Çalgüneri. Rheumatology, Hacettepe University School of Medicine, Ankara, Turkey

\subsection{6/annrheumdis-2001.1013}

Background Acromegalic arthropathy is a noninflammatory condition in which cartilage hypertrophy and hyperplasia lead to the disruption of joint geometry and chondrocyte metabolism, and ultimately to degenerative changes. It is reversible in cases of early and prompt diagnosis. Rheumatoid arthritis is an inflammatory disease with joint destruction. Erosion in rheumatoid arthritis is caused by invasion of articular cartilage by the synovial pannus. Erosion in rheumatoid arthritis may be regressed with early and aggressive treatment. In this report we present a case of acromegaly who developed rheumatoid arthritis.

\section{Objectives}

Methods Case report:A 64-year-old male was admitted in April 2000 for evaluation of a 2-year history of polyarthritis. He was followed in 1985 with symptoms and signs of acromegaly, which was confirmed by radiology and a basal GH level of $30 \mathrm{mU} / \mathrm{l}$. His acromegalic features improved after he had been operated. In 1995, therapy with sandostadin was started. In 1997, he was given external radiation. In 1998, he started to complain symmetric polyarthritis in proximal interphalangial and metacarpophalangial, wrist, and knee joints. In laboratory evaluation, erythrocyte sedimentation rate (ESR) was $93 \mathrm{~mm} /$ hour and rheumatoid factor was $685 \mathrm{IU} / \mathrm{l}$. No improvement was observed with the treatment protocol consisting of non-steroid anti-inflammatory drug and exercise which was given in another hospital. Two years later, he was evaluated in our rheumatology clinic. At that time, he had arthritis in PIF, MCF, and wrist joints and threehour morning stiffness. Bilateral ulnar deviation at MCF joint and interosseous muscle atrophy were observed. In laboratory examination, ESR, CRP, $\mathrm{Hb}, \mathrm{RF}$, platelet count were $89 \mathrm{~mm} /$ hour, $6.7 \mathrm{mg} / \mathrm{dL}, 12.6 \mathrm{gr} / \mathrm{dL}, 789 \mathrm{IU} / \mathrm{L}, 540000 / \mathrm{ml}$ respectively. In radiological evaluation, osteoporosis in lumbar vertebrae and in hand graph were observed. Widening and tufting of distal phalanx and subchondral erosive and cystic changes in PIF and MCF joints were seen. In MRI, synovitis and erosive changes were marked. The patient was diagnosed as rheumatoid arthritis and treated with methotrexate, sulfasalazine, and hydroxychloroquine. After three months of treatment, the patient improved regarding in clinical and laboratory values. He is still being followed by our clinic in good health. 